\title{
WEAKLY CONFLUENT MAPPINGS AND THE COVERING PROPERTY OF HYPERSPACES
}

\author{
J. GRISPOLAKIS AND E. D. TYMCHATYN ${ }^{1}$
}

\begin{abstract}
In this note we characterize the continua which are images of weakly confluent mappings only in terms of a covering property for hyperspaces and in terms on an embedding property. This answers some questions of B. Hughes and A. Lelek.
\end{abstract}

1. Introduction. The notion of Class $(W)$ (i.e., the class of all continua which are images of weakly confluent mappings only) was introduced by A. Lelek in 1972. Since then several authors have studied these continua and have attempted to classify them. For example it has been proved that hereditarily indecomposable continua [1], chainable continua [11] and nonplanar circlelike continua [3] are in Class $(W)$.

In some unpublished work B. Hughes proved that continua with the covering property of hyperspaces are in Class $(W)$ (see [10, (14.73.21)]) and he asked whether the converse is true (see [10, (14.73.25)]). Recently, it was proved that circle-like continua with no local separating subcontinua [4], and tree-like atriodic continua [5] have the covering property, and hence, they are in Class $(W)$. In [5, Theorem 3.1 and Corollary 3.3], a very geometric method was introduced in order to check whether certain classes of continua have the covering property. The following theorem was proved in [4, Theorem 2.2] and it was asked whether its converse is true [4, (5.4)].

1.1. TheOREM [4, TheOREM 2.2]. If $X$ is a continuum which has the covering property, then $X$ is absolutely $C^{*}$-smooth.

The property of being absolutely $C^{*}$-smooth is an embedding property. In particular, if a continuum $X$ is absolutely $C^{*}$-smooth and embeddable in a continuum $Y$, then the embedding has to be so that if $\left\{A_{i}\right\}_{i=1}^{\infty}$ is a sequence of subcontinua of $Y$ converging to $X$ and $B$ is a subcontinuum of $X$ then there exists a sequence of continua $\left\{B_{i}\right\}_{i=1}^{\infty}$ which converges to $B$ and such that $B_{i} \subset A_{i}$ for each $i$.

The purpose of this paper is to resolve all the above-mentioned questions in the affirmative by proving that for an arbitrary continuum being in Class $(W)$, having the covering property, and being absolutely $C^{*}$-smooth are equivalent.

Received by the editors March 5, 1978.

AMS (MOS) subject classifications (1970). Primary 54B20, 54C25, 54C10; Secondary 54C20.

1 The first author was supported by a University of Saskatchewan Postdoctoral Fellowship, and the second author was supported in part by NRC (Canada) grant No. A5616. 
2. Definitions. By a continuum is meant a connected, compact metric space. A mapping is always a continuous function. A mapping $f: X \rightarrow Y$ of a continuum $X$ onto a continuum $Y$ is said to be weakly confluent provided for each subcontinuum $K$ of $Y$ there exists a component $C$ of $f^{-1}(K)$ such that $f(C)=K$. Weakly confluent mappings were first introduced in [8, p. 98] but in fact were used by S. Mazurkiewicz in [9] who proved that essential mappings of compacta onto $n$-cells are weakly confluent. A continuum $X$ is said to be in Class $(W)$ provided that every mapping from any continuum onto $X$ is weakly confluent. Let $A$ be a subset of a metric space $X$ and let $\varepsilon>0$. By $S(A, \varepsilon)$ we denote the $\varepsilon$-ball about $A$. If $X$ is a compact metric space, then the hyperspace $C(X)$ is the space of all nonempty subcontinua of $X$ metrized by the Hausdorff metric $H$, which is defined by

$$
H(A, B)=\inf \{\varepsilon>0 \mid A \subset S(B, \varepsilon) \text { and } B \subset S(A, \varepsilon)\}
$$

for any $A, B \in C(X)$. Let $X$ be a continuum. A Whitney map $\mu: C(X) \rightarrow$ $[0, \infty)$ is a mapping such that $\mu(\{x\})=0$ for each $x \in X$, and such that if $A$, $B \in C(X)$ with $A \subset B \neq A$, then $\mu(A)<\mu(B)$. The existence of such mappings was proved in [12], and the fact that they are monotone was remarked in [2]. We say that a continuum $X$ has the covering property provided that for any Whitney map $\mu$ for $C(X)$ and for any $t \in[0, \mu(X)]$ no proper subcontinuum $\Lambda$ of $\mu^{-1}(t)$ covers $X$ (Krasinkiewicz and Nadler, Whitney Properties, Fund. Math. (to appear)).

Define a function $C^{*}: C(X) \rightarrow C(C(X))$ by $C^{*}(A)=C(A)$ for each $A \in C(X)$. It was proved in $[10,(15.2)]$ that $C^{*}$ is upper semicontinuous. A continuum $X$ is said to be $C^{*}$-smooth at $A, A \in C(X)$, provided that $C^{*}$ is continuous at $A$. The continuum $X$ is said to be $C^{*}$-smooth [10, (15.5)] provided that it is $C^{*}$-smooth at each $A \in C(X)$. We say that a continuum $A$ is absolutely $C^{*}$-smooth at $A$. By Theorem 1.1, we have that tree-like atriodic continua, nonplanar circle-like continua, and hereditarily indecomposable continua are absolutely $C^{*}$-smooth.

3. The Main Theorem. We shall need the following result of Krasinkiewicz $[7,(2.3)]$ :

3.1. Lemma [7, (2.3)]. Let $X$ be a continuum, let $\mu: C(X) \rightarrow[0, \infty)$ be a Whitney map and let $t \in[0, \mu(X)]$. Then for every $\varepsilon>0$ there exists $\eta>0$ such that, for every pair $A, B \in \mu^{-1}(t)$, if $B \subset S(A, \eta)$, then $H(A, B)<\varepsilon$.

3.2. THEOREM. For any continuum $X$ the following are equivalent:

(a) $X$ is in $\operatorname{Class}(W)$,

(b) $X$ has the covering property, and

(c) $X$ is absolutely $C^{*}$-smooth.

Proof. (b) implies (c) by Theorem 1.1.

(c) $\Rightarrow$ (a): Let $X$ be an absolutely $C^{*}$-smooth continuum, and let $f: Y \rightarrow X$ be a mapping of a continuum $Y$ onto $X$. Consider the product space $Y \times I$ of 
$Y$ with the unit interval $I=[0,1]$, and define an equivalence relation $\sim$ on $Y \times I$ by setting $\left(y_{1}, t_{1}\right) \sim\left(y_{2}, t_{2}\right)$ if and only if either $y_{1}=y_{2}$ and $t_{1}=t_{2}$ or $t_{1}=t_{2}=1$ and $f\left(y_{1}\right)=f\left(y_{2}\right)$. Then $\sim$ is upper semicontinuous and the quotient space $Z=(Y \times I) / \sim$ is a continuum. Let $\phi: Y \times I \rightarrow Z$ be the quotient map. Notice that $\phi[Y \times\{1\}]$ is homeomorphic to $X$. We identify $\phi[Y \times\{1\}]$ with $X$. Since $X$ is absolutely $C^{*}$-smooth, we have that the function

$$
C^{*}: C(Z) \rightarrow C(C(Z))
$$

defined by $C^{*}(A)=C(A)$ for each $A \in C(Z)$ is continuous at the point $X$ of $C(Z)$. Let $\left\{t_{i}\right\}_{i=1}^{\infty}$ be a sequence in $[0,1)$ such that $\lim _{i \rightarrow \infty} t_{i}=1$. Then by the continuity of $\phi$ we have that

$$
\operatorname{Lim}_{i \rightarrow \infty} \phi\left[Y \times\left\{t_{i}\right\}\right]=\phi[Y \times\{1\}]=X .
$$

Since $C^{*}$ is continuous at $X$, we have that

$$
\operatorname{Lim}_{i \rightarrow \infty} C\left(\phi\left[Y \times\left\{t_{i}\right\}\right]\right)=C(X) .
$$

By (2), we have that if $K$ is a subcontinuum of $X$, then there exists a sequence $\left\{L_{i}\right\}_{i=1}^{\infty}$ of continua such that $L_{i} \subset \phi\left[Y \times\left\{t_{i}\right\}\right]$ for each $i \in\{1,2, \ldots\}$ and such that

$$
\operatorname{Lim}_{i \rightarrow \infty} L_{i}=K
$$

Since $\phi$ is one-to-one on $Y \times\left\{t_{i}\right\}$ for each $i \in\{1,2, \ldots\}$, there exists a continuum $M_{i}$ in $Y \times\left\{t_{i}\right\}$ such that $\phi\left(M_{i}\right)=L_{i}$ for each $i \in\{1,2, \ldots\}$. We may suppose $M=\operatorname{Lim}_{i \rightarrow \infty} M_{i}$. Then $M$ is a subcontinuum of $Y \times\{1\}$ and $Y \times\{1\}$ is homeomorphic to $Y$. Identify $Y$ with $Y \times\{1\}$. Then, by (3) and the continuity of $\phi$, we infer that $f(M)=K$. This proves that $f$ is weakly confluent, and hence, $X$ is in Class $(W)$.

(a) $\Rightarrow$ (b): Let $X$ be a continuum in Class $(W)$, let $\mu: C(X) \rightarrow[0, \infty)$ be a Whitney map for $C(X)$ and let $\Lambda$ be a subcontinuum of $\mu^{-1}(t)$ for some $t \in[0, \mu(X)]$ such that $\cup \Lambda=X$. We shall prove that $\Lambda=\mu^{-1}(t)$. We may assume, without loss of generality, that $t \in(0, \mu(X))$. Suppose, on the contrary, that there exists $A \in \mu^{-1}(t) \backslash \Lambda$. Then, by Lemma 3.1, there exists some $\eta>0$ such that for each $L \in \Lambda$ we have that $L \not \subset S(A, \eta)$. This implies that for each $L \in \Lambda$ we have that

$$
L \cap[X \backslash S(A, \eta)] \neq \varnothing .
$$

Since $\Lambda$ is compact and $A \notin \Lambda$, we have, by Lemma 3.1 that there exists an integer $m>1$, distinct points $x_{1}, \ldots, x_{m}$ in $A$, and some $0<\varepsilon<\eta$ such that, if $L \in \Lambda$, then $L \cap S\left(x_{i}, \varepsilon\right)=\varnothing$ for some $i \in\{1, \ldots, m\}$.

For each $i \in\{1, \ldots, m\}$ let

$$
\mathfrak{F}_{i}=\left\{L \in \Lambda \mid L \cap S\left(x_{i}, \varepsilon\right)=\varnothing\right\} .
$$

Then each $\mathfrak{F}_{i}$ is a compact subset of $\Lambda$. Let $X_{i}=\cup \mathfrak{F}_{i}$ and let $Y_{i}$ be a space 
homeomorphic to $X_{i}$ such that $Y_{i} \cap Y_{j}=\varnothing$ for each $i \neq j ; i, j \in$ $\{1, \ldots, m\}$. Let $Z$ be the topological sum

$$
Z=Y_{1} \cup \cdots \cup Y_{m}
$$

and let $f: Z \rightarrow X$ be a mapping which carries $Y_{i}$ homeomorphically onto $X_{i}$ for each $i \in\{1, \ldots, m\}$. By [6, p. 23], $X_{i}$ is a compact metric space, and hence, $Y_{i}$ is a compact metric space for each $i \in\{1, \ldots, m\}$. This implies that $Z$ is a compact metric space.

We define an equivalence relation $\sim$ on $Z$ by setting $x \sim y$ if and only if either $x=y$ or $f(x)=f(y)$ and $f(x) \in X \backslash S(A, \eta)$. Then $\sim$ is upper semicontinuous. Let $Y=Z / \sim$ be the quotient space and let $\phi: Z \rightarrow Y$ be the quotient map. Let $g: Y \rightarrow X$ be the unique mapping such that $f=g \circ \phi$. Notice that, since $f$ and $\phi$ are onto, $g$ is also onto. By the construction of $Y$ we have that $g^{-1}(A)$ is the disjoint union of the closed sets

$$
\phi\left[f^{-1}(A) \cap Y_{1}\right], \ldots, \phi\left[f^{-1}(A) \cap Y_{m}\right]
$$

no one of which is mapped by $g$ onto $A$. It remains to show that $Y$ is connected. This will imply that $g: Y \rightarrow X$ is not weakly confluent, contrary to the hypothesis that the continuum $X$ is in $\operatorname{Class}(W)$.

To show that $Y$ is connected, consider the mapping $\hat{g}: C(Y) \rightarrow C(X)$ of $C(Y)$ into $C(X)$ defined by $\hat{g}(K)=g(K)$ for each $K \in C(Y)$. In a similar way define the mappings $\hat{f}: C(Z) \rightarrow C(X)$ and $\hat{\phi}: C(Z) \rightarrow C(Y)$. Notice that $\hat{f}=\hat{g} \circ \hat{\phi}$, and that by the construction of $Z$ and $f$ we have that $\Lambda \subset \hat{f}[C(Z)] \subset C(X)$. We define, now, a set-valued function $F: \Lambda \rightarrow Z$ by

$$
F(L)=\cup\left[\hat{f}^{-1}(L)\right]
$$

for each $L \in \Lambda$. Then $\cup\left[\hat{f}^{-1}(L)\right]$ consists of at most $m$ homeomorphic copies of $L$. Let $G=\phi \circ F$. Then $G: \Lambda \rightarrow Y$ is a set-valued function. Then a simple computation shows that

$$
G(L)=\cup\left[\hat{g}^{-1}(L)\right]
$$

for each $L \in \Lambda$. By the construction of $Y$ and $\phi$ we have that $\cup\left[\hat{g}^{-1}(L)\right]$ is a continuum, and hence, $G$ is a continuum-valued function. Notice that $\cup\{G(L) \mid L \in \Lambda\}=Y$, since $\cup \Lambda=X$. To show that $Y$ is connected, it suffices to show that $G$ is upper semicontinuous. Since $\phi$ is continuous and $G=\phi \circ F$, it is enough to show that $F$ is upper semicontinuous. For this let $\left\{L_{i}\right\}_{i=1}^{\infty}$ be a sequence in $\Lambda$ converging to some $L \in \Lambda$. For each $i \in\{1$, $2, \ldots\}$ and for each $j \in\{1, \ldots, m\}$ let $L_{i}^{j}=\varnothing$ if there does not exist $K \in C\left(Y_{i}\right)$ such that $f(K)=L_{i}$, otherwise, let $L_{i}^{j} \in C\left(Y_{j}\right) \subset C(Z)$ such that $f\left(L_{i}^{j}\right)=L_{i}$. Assume, without loss of generality, that the sequence $\left\{L_{i}^{j}\right\}_{i=1}^{\infty}$ converges to some $L^{j} \in C\left(Y_{j}\right)$ (this is possible since $C\left(Y_{j}\right)$ is compact). By the continuity of $f$, we have that $f\left(L^{j}\right)=L$. Hence, $L^{j} \in$ $\hat{f}^{-1}(L)$ which implies that

$$
L^{j} \subset \cup\left[\hat{f}^{-1}(L)\right]=F(L)
$$


On the other hand, we have that

$$
\operatorname{Lim}_{i} \sup F\left(L_{i}\right)=\operatorname{Lim}_{i} \sup \cup\left[\hat{f}^{-1}\left(L_{i}\right)\right]=\operatorname{Lim}_{i} \sup \bigcup_{j=1}^{m} L_{i}^{j}=\bigcup_{j=1}^{m} L^{j} \text {. }
$$

This together with (4) implies that $\operatorname{Lim}_{\sup _{i}} F\left(L_{i}\right) \subset F(L)$. Thus, $F$ is upper semicontinuous and the theorem is proved.

3.3 REMARK. The fact that in Theorem 3.2 (b) implies (a) was first proved by B. Hughes (see [10, (14.73. 21)]) by using different methods. The equivalence of (a) and (c) seems to be the most satisfactory of all. Finally, we remark that Theorem 3.2 solves some problems of Lelek and Hughes (see [10, (14.73.22)] and [10,(14.73.25)]), and answers in the affirmative questions (5.1), (5.2), (5.3) and (5.4) in [4]. It also generalizes part of Theorem 4.1 in [4], where the equivalence of (a) and (b) was proved for the class of nonchainable circle-like continua.

The following result is an application of Theorem 3.2 and proves the converse of Corollary 3.4 in [5]. Let $Y$ be a subspace of the continuum $X$. Then by $C(Y)$ we denote the subspace of $C(X)$ which consists of all nonempty subcontinua of $Y$.

3.4 Corollary. Let $X$ be a compactification of the half line $[0, \infty)$. Then $X$ is in Class $(W)$ if and only if

$$
C(X)=\mathrm{Cl}[C([0, \infty))]
$$

Proof. Sufficiency was proved in [5, Corollary 3.4]. To prove the necessity of the condition, suppose that $X$ is in Class $(W)$. Let $A_{1} \subset A_{2} \subset \ldots$ be an increasing sequence of arcs in $[0, \infty)$ such that $\operatorname{Lim}_{n \rightarrow \infty} A_{n}=X$. By Theorem 3.2, $X$ is absolutely $C^{*}$-smooth, and hence,

$$
\operatorname{Lim}_{n \rightarrow \infty} C\left(A_{n}\right)=C(X),
$$

which proves the corollary.

Some more applications of Theorem 3.2 and more discussion on Class $(W)$ will be presented in a paper that will follow.

\section{REFERENCES}

1. H. Cook, Continua which admit only the identity mapping onto nondegenerate subcontinua, Fund. Math. 60 (1967), 241-249.

2. C. Eberhart and Sam B. Nadler, Jr., The dimension of certain hyperspaces, Bull. Acad. Polon. Sci. Sér. Sci. Math. Astronom. Phys. 19 (1971), 1027-1034.

3. G. A. Feuerbacher, Weakly chainable circle-like continua, Doctoral Dissertation, Univ. of Houston, Houston, Texas, 1974.

4. J. Grispolakis, Sam B. Nadler, Jr. and E. D. Tymchatyn, Some properties of hyperspaces with applications to continua theory, Canad. J. Math. (to appear).

5. J. Grispolakis and E. D. Tymchatyn, Continua which are images of weakly confluent mappings only, Houston J. Math. (submitted).

6. J. L. Kelly, Hyperspaces of a continuum, Trans. Amer. Math. Soc. 52 (1942), 22-36.

7. J. Krasinkiewicz, On the hyperspaces of snake-like and circle-like continua, Fund. Math. 83 (1974), 155-164. 
8. A. Lelek, A classification of mappings pertinent to curve theory, Proceedings of the Oklahoma Topology Conference, Univ. of Oklahoma, Norman, Okla., 1972, pp. 97-103.

9. S. Mazurkiewicz, Sur l'existence des continues indécomposables, Fund. Math. 25 (1935), 327-328.

10. Sam B. Nadler, Jr., Hyperspaces of sets, Dekker, New York, 1978.

11. D. R. Read, Confluent and related mappings, Colloq. Math. 29 (1974), 233-239.

12. H. Whitney, Regular families of curves. I, Proc. Nat. Acad. Sci. U.S.A. 18 (1932), 275-278.

Department of Mathematics, University of Saskatchewan, Saskatoon, Saskatchewan, CANADA S7N OWO 